\title{
Carpal Tunnel Syndrome Caused by High Division of the Median Nerve Proximal to the Carpal Tunnel: A Case Report
}

\author{
Yeo-song Kim ${ }^{1}$, Hak-cheol $\mathrm{Ko}^{1}$, Byung-chul Son ${ }^{1,2}$ \\ ${ }^{1}$ Department of Neurosurgery, Seoul St. Mary's Hospital, College of Medicine, The Catholic University of Korea, Seoul, \\ ${ }^{2}$ Catholic Neuroscience Institute, College of Medicine, The Catholic University of Korea, Seoul, Republic of Korea
}

Corresponding author: Byung-chul Son

Department of Neurosurgery,

Seoul St. Mary's Hospital,

Catholic Neuroscience Institute, College of Medicine, The Catholic University of Korea, 222 Banpo-daero, Seocho-gu, Seoul 06591, Republic of Korea Tel: +82-2-2258-6122

Fax: +82-2-594-4248

E-mail: sbc@catholic.ac.kr

Received: February 9, 2018 Revised: March 13, 2018 Accepted: March 22, 2018
High division of the median nerve proximal to the carpal tunnel (known as a bifid median nerve) is a median nerve anomaly with incidence rate of $2.8 \%$. This rare entity is often associated with persistent median artery, aberrant muscles, and carpal tunnel syndrome (CTS). Bifid median nerve is a cause of secondary CTS due to its relatively higher cross-sectional area compared to a non-bifid median nerve. We report a case of median nerve entrapment at the left wrist associated with bifid median nerve and ramification of a persistent median artery. A 69-year-old, right-handed woman, without noticeable medical history, presented chronic paresthesia in her left hand and fingers with duration of two years. Magnetic resonance imaging (MRI) of the left wrist showed a swollen, enlarged bifid median nerve proximal to the flexor retinaculum. MRI revealed a typical bifid median nerve proximal to the carpal tunnel. Decompression of the median nerve was carefully performed with extended forearm incision. Median nerve variation should be considered when performing open or endoscopic carpal tunnel release to prevent iatrogenic injuries.

Key Words: Carpal tunnel syndrome; Congenital abnormalities; Magnetic resonance imaging; Median nerve

\section{INTRODUCTION}

Carpal tunnel syndrome (CTS) is a common neuropathy caused by entrapment of the median nerve by a thickened flexor retinaculum in the wrist ${ }^{4,9,14)}$. However, various causes of secondary CTS have been reported, including vascular anomalies involving persistent median artery, variations of the median nerve, and space-occupying lesions in the wrist and palm ${ }^{14,7-9,13)}$. High division of the median nerve proximal to the carpal tunnel (known as a bifid median nerve) is a median nerve anomaly with incidence rate of $2.8 \% \%^{7,9)}$. Bifidity of the median nerve is known to predispose the median nerve to compression because the relatively higher cross-sectional area of the 2 nerve bundles ${ }^{3,5,15)}$.

Although the current authors do not routinely request magnetic resonance imaging (MRI) for the diagnosis of CTS, high bifurcation of the median nerve was diagnosed with preoperative imaging study for long-standing paresthesia in the nondominant hand. By direct visualization of the swollen, bifid median nerve proximal to the carpal tunnel, we could prevent an occurrence of inadvertent median nerve injury and incomplete decompression of the median nerve.

\section{CASE REPORT}

A 71-year-old right-handed female patient presented with a 2-year history of gradually worsening paresthesias, numbness, and tingling in the lateral three digits and radial palm in her left hand. There was no constant aching pain in the radial palm. No posterior neck pain or radicular symptoms in her left arm was evident. The paresthesia insidiously developed after 3-month exercise of keyboard typing. It was present day and night with no typical feature of waking paresthesia in the night and did not show an aggravation despite of daytime use of the hands. Her medical history was unremarkable including diabetes and hypothyroidism. However, she had underwent an operation for CTS in her right wrist 15 years ago in an another hospital with almost similar symptoms of the current presentation. The operation was successful. No paresthesia or hand weakness remained thereafter in her right hand and fingers.

Physical examination showed mild wasting of the left thenar muscles. However, weakness in the opposition, flexion, and palmar abduction of the thumb was not observed. Sensation to light touch and pinprick was intact. Tinel sign was not 
elicited. However, a positive Phalen's test was present on the left wrist. Paresthesia in the left hand initially responded to medication and local steroid injection. However, continuous paresthesia progressively worsened over time, eventually showed no response to conservative treatment 6 months prior to admission. Electrodiagnostic findings were compatible with median entrapment neuropathy around the right wrist and clinical CTS with mild to moderate degree by American Association of Electrodiagnostic Medicine (AAEM) classification ${ }^{14)}$. Considering her chronic, continuous paresthesia without aggravation of symptom at night despite of daytime hand use, an MRI was requested. MRI revealed a bilobed appearance of swollen bifid median nerve in distal forearm proximal to the flexor retinaculum (Fig. 1). The median nerve in the carpal tunnel at the level of the pisiform bone was also enlarged (Fig. 1A). The cross-sectional area of the median nerve at that level was $15.07 \mathrm{~mm}^{2}$ (ImageJ; National Institute of Health, Bethesda, MD,
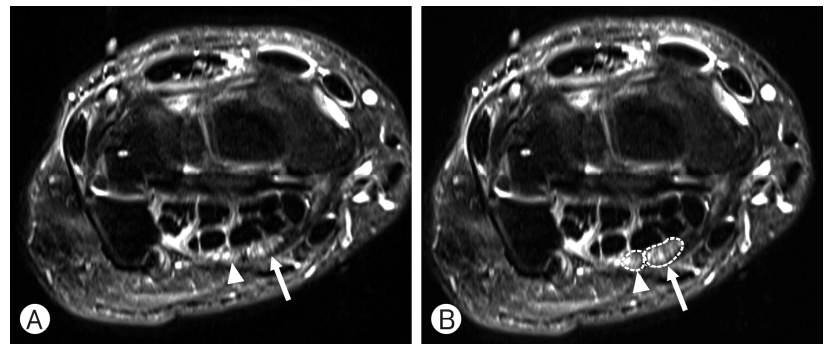

Fig. 1. High division of the median nerve proximal to the carpal tunnel (a bifid median nerve). (A) A T2-weighted axial, fat suppression magnetic resonance imaging of the left wrist (left) showing a bilobed median nerve at the level of the pisiform bone, just proximal to the flexor retinaculum in the distal forearm. Note the radial (arrow) and ulnar (arrowhead) divisions of the median nerve. (B) Measurement of the cross-sectional area of both radial (arrow) and ulnar (arrowhead) divisions of bifid median nerve at the level of the pisiform bone. The cross-sectional area of the median nerve at that level was $15.07 \mathrm{~mm}^{2}$.

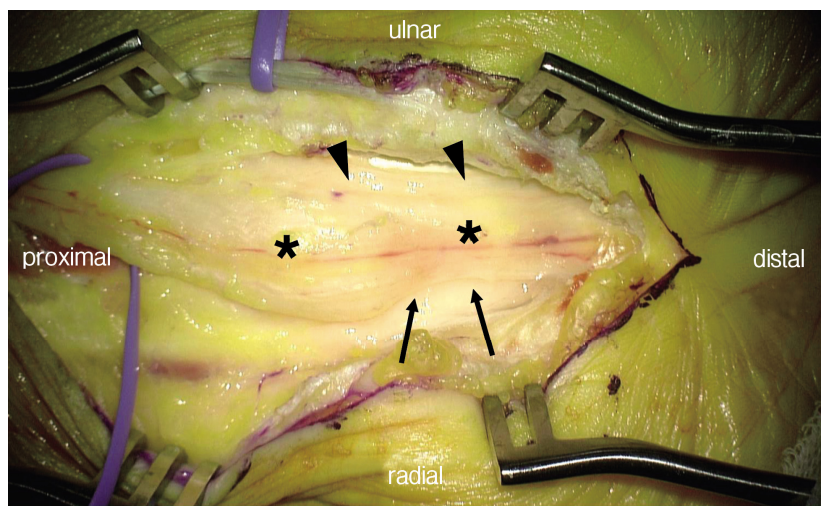

Fig. 2. An intra-operative photography showing the swollen radial (black arrowheads) and ulnar (black asterisks) divisions of the bifid median nerve in the distal forearm proximal to the flexor retinaculum. Actual compression site was proximal to the carpal tunnel (black arrows).
USA) (Fig. 1B). There was no abnormal signal intensity or gadolinium enhancement along the course of the median nerve in the MRI.

After incision of the skin and superficial fascia of the distal forearm and the palm, the underlying flexor retinaculum was identified and transected. The forearm fascia was then carefully dissected. The underlying median nerve just proximal to the flexor retinaculum in distal forearm was found to be duplicated and enlarged (Fig. 2). After dissection of the subparaneurial space, the adhesion between the radial and medial division of the bifid median nerve was detached carefully. The entire course of bifid median nerve was mobilized free from distal forearm to the carpal tunnel. Persistent paresthesia in radial palm and fingers improved immediately after the operation. Postoperative course was uneventful. Minimal dysesthesia without any paresthesia in the distal portion of the radial three digits remained until 6-month follow-up at an outpatient clinic.

\section{DISCUSSION}

\section{Anomaly of the Median Nerve as a Cause of Secondary CTS}

Primary CTS may result from any pathologic condition that causes either reduction in size of the carpal tunnel or an increase in its content because of inflammatory or degenerative changes. However, diverse causes of secondary CTS including anatomic variations of the median nerve within the carpal tunnel have been described. A bifid median nerve is a type of various anomaly and it occurs relatively frequently in patients with CTS $^{(6)}$. The bifid nature may facilitate compression in the carpal tunnel because of its higher cross-sectional area compared with a non-bifid median nerve ${ }^{5,15)}$. According to the classification by Lanz ${ }^{9}$, it is type III anomaly involving the median nerve in the carpal tunnel and its incidence was 2.8\%. Persistent median arteries has been reported to be usually accompanied bifid median nerves". Although persistent median artery was not found intraoperatively in the current case, a fine streak of ramified vascular structure was evident (Fig. 1C). The 2 parts of bifid median nerve may be equal in size ${ }^{9)}$, or a predominance may exist of either the radial or ulnar part ${ }^{1,3)}$. Larger radial division approximately equal in size to a normal median nerve ${ }^{1)}$. In the current case, the radial division of bifid median nerve was much bigger in size and a ramified streak of persistent median vessel was located in the radial one.

Variations involving the median nerve should be considered when performing open or endoscopic carpal tunnel release to prevent iatrogenic injuries ${ }^{3)}$. Inadvertent injury to the median nerve during carpal tunnel surgery can be minimized if the variations are recognized. Although it has no electrophysiological or clinical differential diagnosis, physicians should consider the possibility of median nerve variation in patients with unilateral severe CTS, especially in the nondominant hand ${ }^{3)}$. 


\section{Imaging Study as Preoperative Evaluation of CTS}

The diagnosis of CTS is based on the patient's clinical history and presentation, physical examination, and electrophysiological studies. During the last decade, MRI and ultrasonography have been proven a useful diagnostic tool in patients with $\mathrm{CTS}^{3,5,10,11)}$. The current authors do not routinely use MRI for the diagnosis of CTS. We have requested MRI examination in cases with suspicious secondary causes: relatively young age, male sex, and unilateral occurrence ${ }^{3,12,16,17)}$. MRI was performed in the current case for unremitting, continuous paresthesia without nighttime aggravation of symptoms. The MRI revealed high division of the median nerve proximal to the carpal tunnel (bifid median nerve).

MRI is an already established, effective imaging method to evaluate disorders of the wrist including CTS ${ }^{6,10,15)}$. Criteria for diagnosing CTS on MRI include swelling of the median nerve proximal to the carpal tunnel, and palmar bowing of the flexor retinaculum $^{11,15)}$. Although swelling of the median nerve proximal to the carpal tunnel is considered as reliable diagnostic criteria of CTS, its swelling within the carpal tunnel is not $\operatorname{diagnostic}^{10)}$. In a quantitative MRI study of CTS $^{11)}$, the only statistically significant difference found between patients with CTS and asymptomatic subjects was swelling (>50\% larger in the cross-sectional area) of the median nerve proximal to the carpal tunnel. There was no significant difference in the crosssectional area of the nerve within the carpal tunnel compartment, the size of the tunnel, and flattening of the nerve on entering the carpal tunnel ${ }^{11)}$. Another significant difference between 2 groups was palmar bowing of the flexor retinaculum only at the level of the hamate ${ }^{11)}$. No correlation was found between the electrodiagnostic studies and the parameters of MRI finding for CTS ${ }^{11}$.

Although both ultrasonography and MRI are known to be effective in diagnosis and delineation of a bifid median nerve in the wrist, there was a controversy in the role of ultrasonography for evaluating bifid median nerves. Propeck et al. ${ }^{15)}$ described that the ultrasonographic size criteria for diagnosing CTS in non-bifid median nerves may not accurate in evaluating bifid median nerves. A recent ultrasonographic study" of axial sonograms of 320 hands of 170 patients with CTS and 240 hands of 120 unaffected individuals showed that the cross-sectional area at the level of pisiform were slightly higher than those with a non-bifid median nerve. The cutoff values for the cross-sectional area at the level of the pisiform were $0.11 \mathrm{~cm}^{2}$ (sensitivity, 90\%; specificity, 99\%; $\mathrm{p}<0.001$ ) for patients with a bifid median nerve and $0.10 \mathrm{~cm}^{2}$ (sensitivity, 98\%; specificity, $81 \%$; $\mathrm{p}<0.001$ ) for patients with a non-bifid median nerve ${ }^{5}$. Although ultrasonography is an effective means of evaluation of structural abnormalities in patients with CTS, the difference in the cross-sectional area between bifid and non-bifid median nerves was not great. MRI seems to be more useful than ultra- sonography in displaying the carpal tunnel and its contents, including the median nerve, with conspicuity ${ }^{15)}$. Similarly, MRI facilitates the detection of anatomical variations in and around the carpal tunnel ${ }^{15)}$.

\section{CONCLUSION}

The authors experienced a rare occurrence of high-division of the median nerve proximal to the carpal tunnel in the nondominant hand in a patient with chronic disabling paresthesia. Inadvertent injury to the median nerve could have been avoided with preoperative MRI examination.

\section{CONFLICTS OF INTEREST}

No potential conflict of interest relevant to this article was reported.

\section{REFERENCES}

1. Al-Qattan MM, Al-Zahrani K, Al-Omawi M: The bifid median nerve re-visited. J Hand Surg Eur Vol 34:212-214, 2009

2. Amadio PC: Anatomical variations of the median nerve within carpal tunnel. Clin Anat 1:23-31, 1988

3. Bagatur AE, Yalcinkaya M, Atca AO: Bifid median nerve causing carpal tunnel syndrome: MRI and surgical correlation. Orthopedics 36:e451-e456, 2013

4. Barfred T, Højlund AP, Bertheussen K: Median artery in carpal tunnel syndrome. J Hand Surg Am 10:864-867, 1985

5. Bayrak IK, Bayrak AO, Kale M, Turker H, Diren B: Bifid median nerve in patients with carpal tunnel syndrome. J Ultrasound Med 27:1129-1136, 2008

6. Davis TR: Diagnostic criteria for upper limb disorders in epidemiological studies. J Hand Surg Br 23:567-569, 1998

7. Davlin LB, Aulicino PL, Bergfield TL: Anatomical variations of the median nerve at the wrist. Orthop Rev 21:955-959, 1992

8. Jang SR, Choi JG, Son BC: Compression of the median nerve by a lipoma in the distal forearm associated with bilateral Carpal tunnel syndromes. Nerve 2:84-86, 2016

9. Lanz U: Anatomical variations of the median nerve in the carpal tunnel. J Hand Surg Am 2:44-53, 1977

10. Mesgarzadeh M, Schneck CD, Bonakdarpour A, Mitra A, Conaway D: Carpal tunnel: MR imaging. Part II. Carpal tunnel syndrome. Radiology 171:749-754, 1989

11. Monagle K, Dai G, Chu A, Burnham RS, Snyder RE: Quantitative MR imaging of carpal tunnel syndrome. AJR Am J Roentgenol 172:1581-1586, 1999

12. Nakamichi K, Tachibana S: Unilateral carpal tunnel syndrome and space-occupying lesions. J Hand Surg Br 18:748-749, 1993

13. Park JJ, Choi JG, Son BC: Carpal tunnel syndrome caused by bifid median nerve in association with anomalous course of the flexor digitorum superficialis muscle at the wrist. Nerve 3:2123, 2017 
14. Preston DC, Shapiro BE: Electromyography and neuromuscular disorders: Clinical electrophysiologic correlations, ed 2nd. Philadelphia, PA: Elsevier, 2005, pp255-280

15. Propeck T, Quinn TJ, Jacobson JA, Paulino AF, Habra G, Darian VB: Sonography and MR imaging of bifid median nerve with anatomic and histologic correlation. AJR Am J Roentgenol 175:
1721-1725, 2000

16. Sipos DA: Carpal tunnel syndrome. Orthop Nurs 14:17-20, 1995

17. Treaster DE, Burr D: Gender differences in prevalence of upper extremity musculoskeletal disorders. Ergonomics 47:495-526, 2004 